\title{
MALDI-TOF INVESTIGATION OF LYSOZYME-ALBUMIN INTERACTION
}

\author{
Marharyta Vasylieva, Taras Gromovoy* \\ O. Chuiko Institute of Surface Chemistry, National Academy of Sciences of Ukraine, \\ 17. General Naumov str., Kyiv 03164, Ukraine \\ "e-mail: gromovoy@mail.md,phone: (+38 044)4249456
}

\begin{abstract}
Proteins are the main components of living systems therefore they are a subject of study from different points of view. Under certain conditions, proteins are capable of self-organization forming oligomeric structures of various composition and new properties. The investigations of these transformations were carried out on two well known proteins: albumin and lyzozyme. It is shown that with increase in the concentration of lysozyme in relation to that of albumin the intensities of the peaks corresponding to associates, related to the intensities of the peaks corresponding to albumin and lysozyme, are also increased. Associates of albumin and lysozyme are forming in the stoichiometric ratios of 1:1, 1:2, 1:3, 1:4. A similar pattern is observed in the case of albumin dimer.
\end{abstract}

Keywords: MALDI-TOF, albumin, lysozyme, protein-protein interaction.

\section{Introduction}

Proteins, as the main components of living systems, are an interesting object of study from different points of view. At the molecular level, with application of new physical and chemical methods, research on some aspects of the evolution of matter and the nature of the interaction of macromolecules with each other has become available [1]. It is known that proteins are capable of self-organization outside the living system. They form oligomeric structures of various composition new properties [2-4].

The study of self-organization of proteins is carried out by different methods and in different systems. This can be a chromatographic separation of the associates, changes in the intensity of scattered light, sedimentation analysis, IR spectroscopy, changing $\mathrm{pH}$ etc. [5]. All these methods indicate the emergence of new properties of associates, but quantitative characteristics of mentioned associates are unexplored, such as is the nature of quantitative and qualitative changes of associates formed at different concentrations of proteins in solution [6,7]. The important issue in this research is preserving of the native structure of proteins, which suggests the possibility of these processes to take place in vivo [8,9].

In the present study proteins albumin and lysozyme were selected, due to their simultaneous presence in biological fluids and their functions. Lysozyme and albumin are found in the contact with the environment - the mucous membrane of the nasopharynx, oral and gastrointestinal tract, lacrimal fluid. Lysozyme belongs to the class of hydrolases that destroy the cell walls of bacteria. Albumin belongs to a class of transport proteins that exhibit high binding effect to low-molecular-weight compounds and contains both hydrophilic and lipophilic areas. Also, the intensity of inflammation in vivo may vary with the strength of binding proteases (antimicrobial proteins) by albumin.

The study of such systems can be considered from different points: as a component of biological fluids (changes in the quantitative composition and properties) used as the base or the active ingredient in the pharmaceutical industry, the determination of constants of interaction with other macromolecules, modeling of protein- protein and protein-ligand (complex formation) interactions [10,11].

\section{Experimental}

Lysozyme and human serum albumin (HSA) were dissolved in water at concentration of $1 \mathrm{mg} / \mathrm{mL}$. Lysozyme solution was mixed with a solution of HSA in different ratios. Solution 1 consisted of $0.1 \mathrm{~mL}$ HSA and $0.4 \mathrm{~mL}$ of lysozyme, solution 2 - $0.2 \mathrm{~mL}$ HSA and $0.3 \mathrm{~mL}$ of lysozyme, solution $3-0.3 \mathrm{~mL}$ HSA and $0.2 \mathrm{~mL}$ lysozyme, and solution $4-0.4 \mathrm{~mL}$ HSA and $0.1 \mathrm{~mL}$ of lysozyme. After incubation under standard conditions for $2 \mathrm{~h}$ solutions were investigated by MALDI-TOF. We used this approach to mixing in order to maintain a uniform concentration of proteins in the mixture. This preserves the matrix to analyte ratio in all cases, which increases the reliability of the mass spectra and enables a comparative analysis.

\section{Results and discussion}

In the Figure 1, the mass spectra of the starting compounds are presented. As Figure 1 shows, albumin (m/z 66000) forms homogenous dimers $(\mathrm{m} / \mathrm{z} 132,000)$ and trimers $(\mathrm{m} / \mathrm{z} 198000)$ under the conditions of our experiment. Lysozyme $(\mathrm{m} / \mathrm{z} 14200)$ under these conditions forms homooligomers with high oligomerization number up to 5 (Figure 1).

For solutions 1-4 in all the mass spectra, the formation of heterocompositions of albumin and lysozyme with different stoichiometric ratios was observed (Figure 2). The solution 1 is characterized by presence of the heterostructures albumin - lysozyme and albumin dimer - lysozyme at stoichiometric ratios of (1:1, 1:2) and (1:1), respectively. With advance of lysozyme concentration relative to that of albumin (solution 2), peaks observed in the mass spectrum 
correspond to the formation of the above-mentioned products along with (albumin - lysozyme) complex with ratio (1:3). With further increase in the concentration of lysozyme (solutions 3 and 4), the formation of the structures (albumin lysozyme dimer), ratio 1:4 and (albumin - lysozyme), ratio 1:2 takes place. In all cases, we observed no changes in the values of $\mathrm{m} / \mathrm{z}$ for peaks of lysozyme, albumin and their homo structures. Experimentally obtained values of $\mathrm{m} / \mathrm{z}$ of the hetero structures correspond to the theoretically calculated ones. General trends in the studied systems are as follows: with increasing of lysozyme concentration relative to albumin not only qualitative changes of the mix composition are observed, but an increase in the relative intensities of the mass spectrometric peaks belonging to hetero structures (related to the intensities of peaks of albumin and its dimer) is also detected. These changes indicate a) the formation of new structures and b) the increase of their concentration in solution with the increase of lysozyme / albumin ratio in solution.

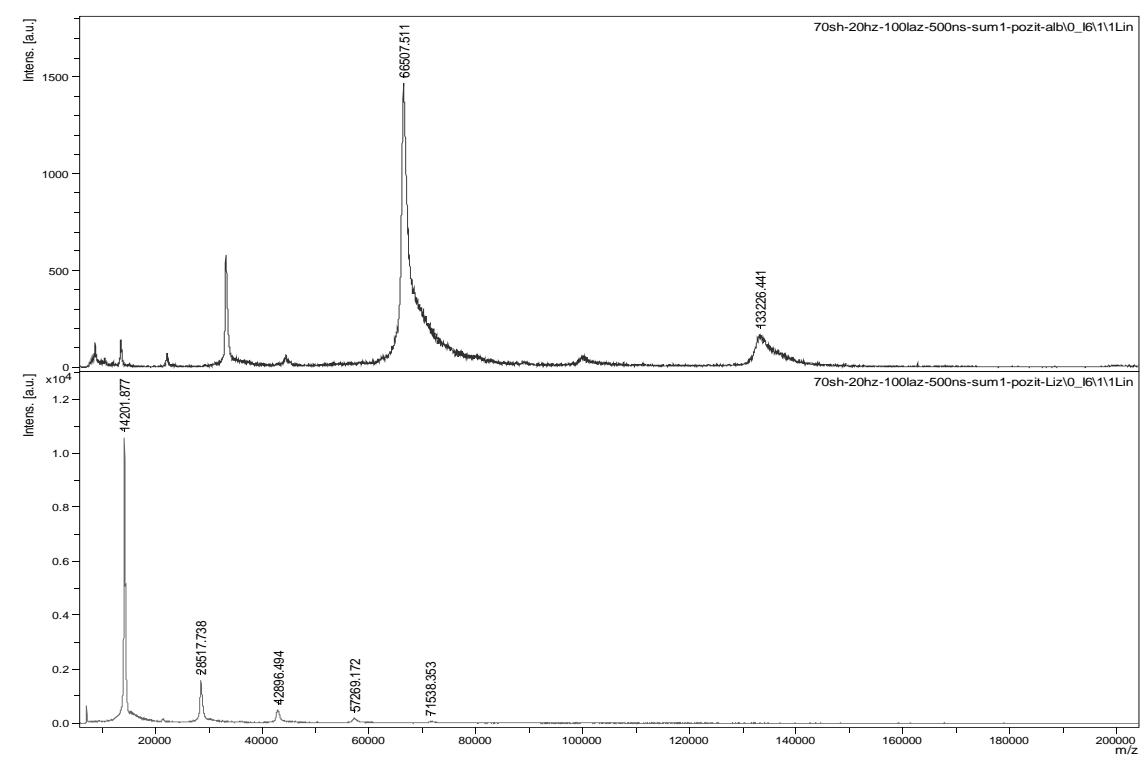

Figure 1. Mass spectra of individual substances albumin (top) and lysozyme (bottom).

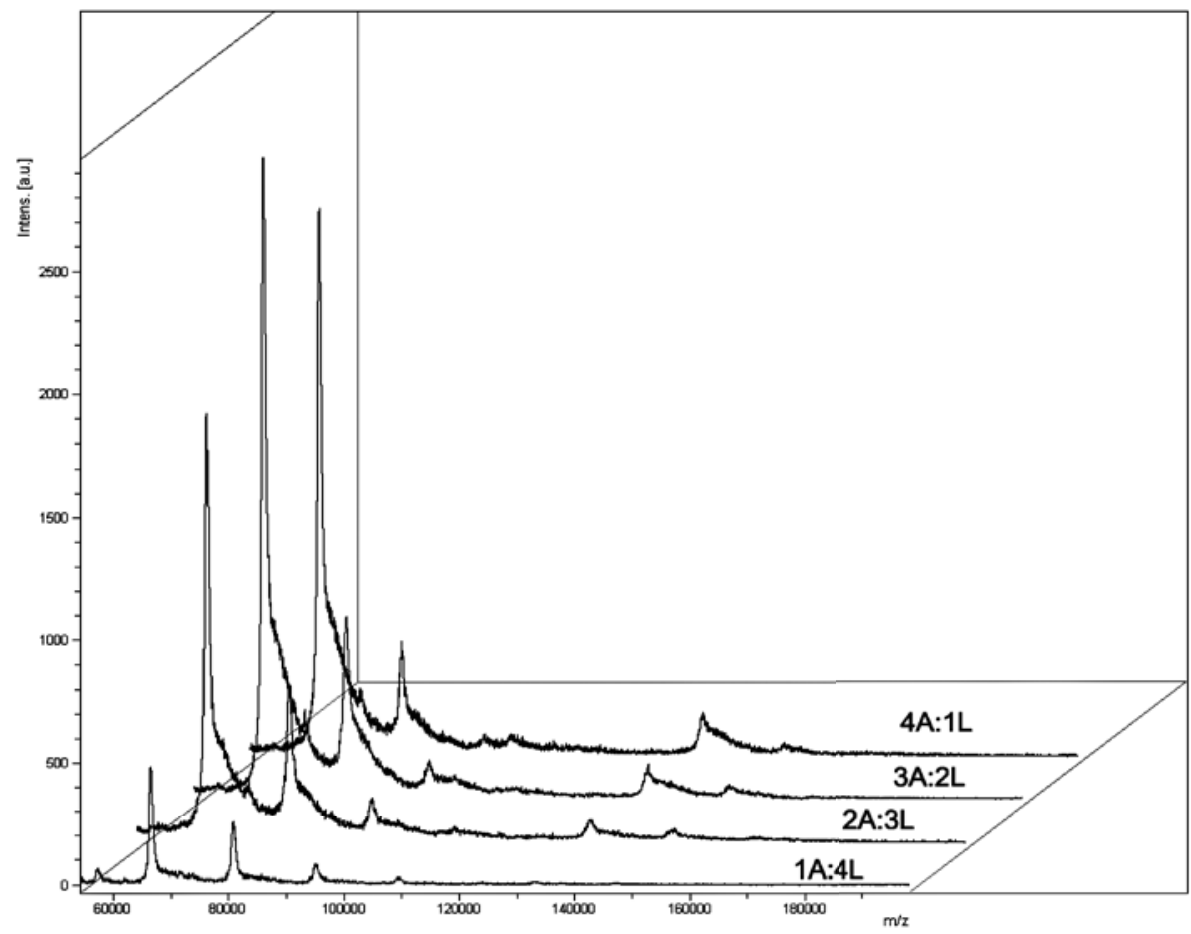

Figure 2. MALDI-ToF mass spectra of solutions 1-4. 
In this paper, an attempt is made to evaluate the qualitative changes in the dynamics of heterostructures formation upon protein concentration. As an option, which corresponds to the dynamics of change in the systems, we have chosen the ratio of the relative intensities of the corresponding hetero structures in the mass spectrum for that of albumin and its dimer.

Description of these curves was performed according to the equation:

$$
y=a \frac{k x^{n}}{1+k x^{n}},
$$

where,

$x$ - the ratio of the concentration of lysozyme to albumin in solution;

$k$ - apparent stability constant of the certain composition;

$a, n$ - empirical coefficients.
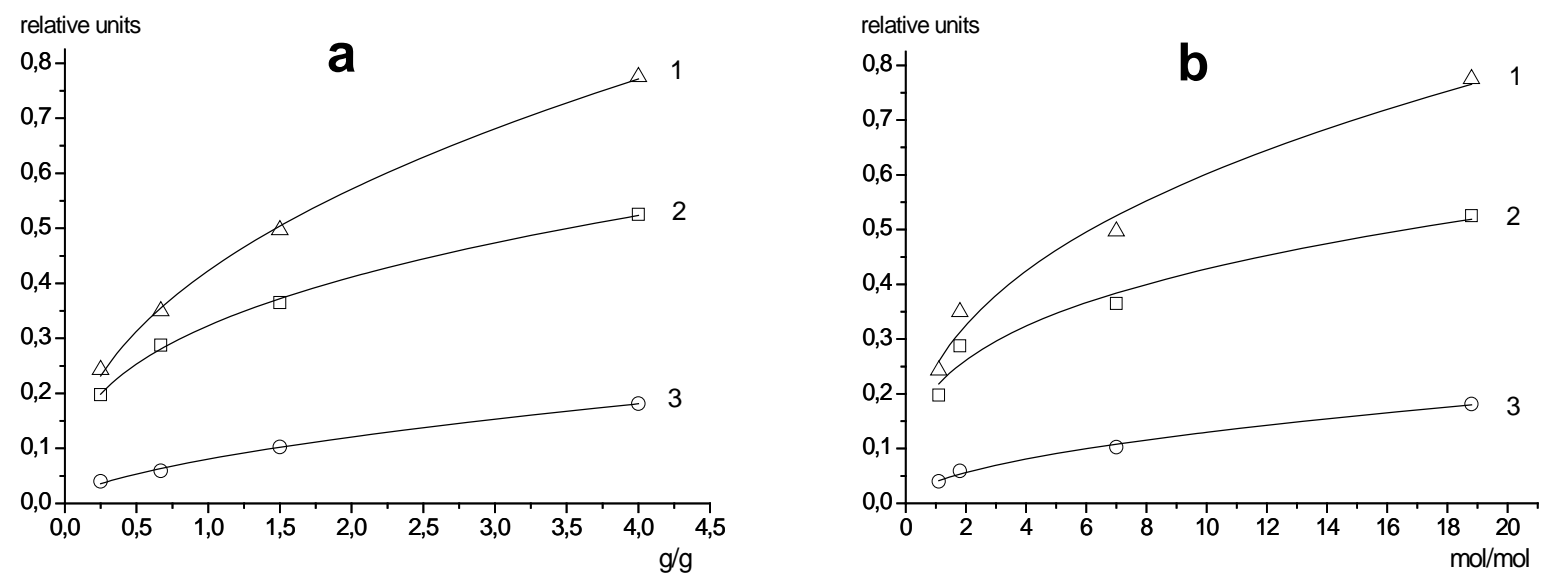

Figure 3. Relative content of albumin and lysozyme oligomers depending on mass (a) and molecular (b) concentration.

1- $\left(\mathbf{A L}+\mathbf{A L}_{2}\right) / \mathbf{A}$

2- AL/A

3- $\mathrm{AL}_{2} / \mathrm{A}$

The calculated constants are characteristic (reflecting only a trend in the system). Their value may differ from the actual stability constants of associates (see Figure 3). For each system the stability row of the compounds (by the value of $k$ ) is evaluated and listed in the Table 1.

It should be noted that the formation of equal weight oligomers can occur in different ways. This was illustrated by the associate $\mathrm{AL}_{2}$.

Table 1

Apparent stability constant of the oligomers.

\begin{tabular}{ccc}
\hline Oligomeric forms & $g / g$ & $\mathrm{~mol} / \mathrm{mol}$ \\
\hline $\mathrm{A}_{2} \mathrm{~L}$ & $2.85 \mathrm{E}+00$ & $0.94 \mathrm{E}+00$ \\
$\mathrm{AL}_{2}$ & $2.80 \mathrm{E}-05$ & $2.69 \mathrm{E}-08$ \\
$\mathrm{AL}$ & $3.70 \mathrm{E}-06$ & $4.70 \mathrm{E}-09$ \\
\hline
\end{tabular}

For $\mathrm{AL}_{2}$ formation we consider two mechanisms (Figure 4). It is logical to assume that an increase in the content of lysozyme in solution leads to the increase in the content of its dimer. But Figure 4 shows that the content of lysozyme dimer with increase of the lysozyme concentration passes through a maximum, indicating that $\mathrm{AL}_{2}$ formation is more likely to occur by the second mechanism: the albumin molecule joins the molecule of lysozyme dimer. 


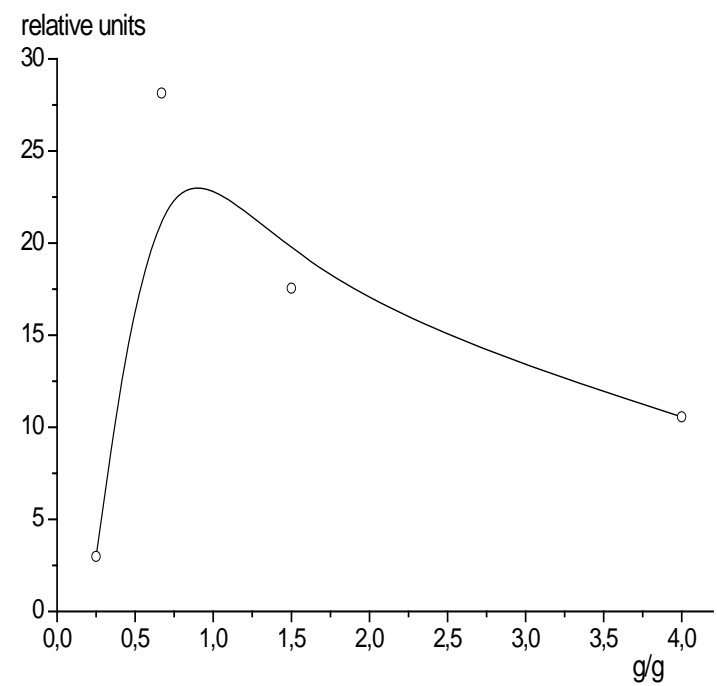

Figure 4. Relative content of lisozyme dimer in solutions versus concentration.

\section{Conclusions}

It has been shown that the oligomerization processes of albumin and lysozyme can be studied by means of MALDI-TOF mass spectrometry. E. g., the mass spectrometric peaks corresponding to the formation of associates (albumin - lisozyme) in the stoichiometric ratios of 1:1, 1:2, 1:3, 1:4, also associates (lysozyme dimer - albumin) in the stoichiometric ratios of 1:1, 1:2.

It has been demonstrated that with increase in the concentration of lysozyme in relation to that of albumin the intensities of the peaks corresponding to associates, related to the intensities of the peaks corresponding to albumin and lysozyme, are also increased. A similar pattern is observed in the case of albumin dimer.

Usage of a modified Freundlich equation for mathematical description of the associates formation processes in albumin-lisozyme solution has been proposed, as the experimental data are well described by this equation.

The stability rows of the associates have been found based on the value of the apparent stability constants. It has been shown that the stability of $A L$ complex is higher than that of $A_{2} L$ and $A_{2}$ complexes.

\section{References}

1. Rapis, E. To the problem of nucleation (the formation of cells) with nanostructures self protein in vitro and in vivo. Journal of Technical Chemistry, 2005, 75(6), pp. 101-113 (in Russian).

2. Nishikawa, T.; Akiyoshi, K.; Sunamoto, J. Macromolecular Complexation between Bovine Serum Albumin and the Self-Assembled Hydrogel Nanoparticle of Hydrophobized Polysaccharides. Journal of the American Chemical Society, 1996, 118(26), pp. 6110-6115.

3. Rapis, E. Evolutionary aspect of protein folding. Journal of Technical Chemistry, 2008, 78(6), pp. 110-115 (in Russian).

4. Rapis, E. On the nonequilibrium phase transition protein. Journal of Technical Chemistry, 2007, 77(6), pp. 109-115 (in Russian).

5. Yeh, P.-R.; Tseng, W.-L. Human serum albumin-coated gold nanoparticles for selective extraction of lysozyme from real-world samples prior to capillary electrophoresis. Journal of Chromatography A, 2012, 1268, pp.166-172.

6. Reynolds, C.; Damerell, D.; Jones, S. ProtorP: a protein-protein interaction analysis server. Structural bioinformatics, 2009, 25(3), pp. 413-414.

7. Almeida, N.L.; Oliveira, C.L.P.; Torriani, I.L.; Loh, W. Calorimetric and structural investigation of the interaction of lysozyme and bovine serum albumin with poly(ethylene oxide) and its copolymers. Colloids and Surfaces B: Biointerfaces, 2004, 38, pp. 67-76.

8. Steiner, R.F. Reversible Association Processes of Globular Proteins. II. Electrostatic Complexes of Plasma Albumin and Lysozyme. Global Proteins II, pp. 56-75.

9. Steiner, R.F. Reversible Association Processes of Globular Proteins. IV. Fluorescence Methods in Studying Protein Interactions. Global Proteins, IV, pp. 291-311.

10. Schreiber, G. Kinetic studies of protein-protein interactions. Current Opinion in Structural Biology, 2002, 12, pp. 41-47.

11. Singh, M. Structural Interactions of Globular Proteins-Bovine Serum Albumin, Egg Albumin, and Lysozyme, in Aqueous Medium, Elucidated with Molar Volumes, Viscosities, Energy Functions, and IR Spectra from 293.15 to 303.15 K. Journal of Applied Polymer Science, 2007, 103, pp. 1420-1429. 